From the Dopartment of Human Geneties, The University of Michigan Medical School, Ann Arbor, Michigan

\title{
Quantitative Variation of the A Antigen at Birth: Its Significance in ABO Hemolytic Disease and in the Infant's Development
}

\author{
by F. J. GRUNDBACHER
}

ABO hemolytic disease of the newborn poses certain prognostic problems because maternal antibody titer is poorly related to severity of the ensuing hemolytic process. Heterogeneity of anti-A antibodies, as recently discussed by Polley et al. [10], may be one of the factors responsible for this poor association. Another factor may be the quantitative behavior of the $A$ antigen. Infants with ABO hemolytic disease are predominantly of subtype $A_{1}$ and not $A_{2}[5,15]$, and the $A$ antigen is "stronger" in the $A_{1}$ than the $A_{2}$ subtype. Furthermore, the A antigen of erythrocytes undergoes marked quantitative change during life; for example, in embryonic erythrocytes it is initially weak but increases slowly during fetal life [8]. After birth, the strength of the $A$ antigen increases rapidly during the first few months of life and then at a slower rate until adult values are reached at about 3 years of age or shortly thereafter [4]. Among adults there is still considerable variation within the A subtypes, which is partly

This work was supported in part by the United States Public Health Service, National Institutes of Health fellowship GPD-15, 166, and in part by grant AT( 1 1.1) 405 from the United States Atomic Energy Commission. hereditary; this latter aspect will be discussed in a forthcoming publication.

Studies have been conducted to evaluate the strength of the A antigen of erythrocytes as a factor in the etiology of ABO hemolytic disease of the newborn. A previous investigation was concerned with the determination of the strength of the A antigen in families ascertained for having one or more type A infants suffering from ABO hemolytic disease [5]. In 31 such families there were 47 infants with this disease, 45 were subtype $A_{1}$ while 2 were $A_{2}$, i.e., a highly significant excess of $A_{1}$ over $A_{2}$. However, antigen strength of the type $A_{1}$ fathers was not significantly different from males of similar ages in the general population. The present communication provides data on the amount and the sources of variability in the strength of the $A$ antigen at birth.

\section{Materials and Methods}

Fresh samples of blood from the umbilical cord of infants born to type A mothers were collected in the Maternity Clinic of the University Hospital in Ann Arbor, Michigan. If the cord blood was type $A$, a fresh blood sample was also taken from the mother, 


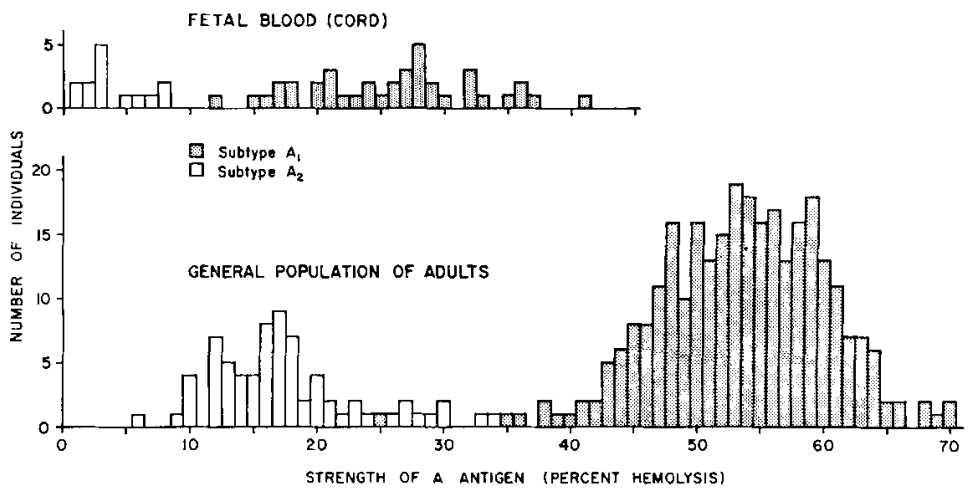

Fig. 1. Frequency distribution of antigen strength among newborn infants and "adults" (6 years of age and older).

whenever possible. Mother-infant pairs both of type A lessened the probability of the presence of maternal anti-A on fetal erythrocytes, as is likely the case among $A$ infants born to type $O$ and $B$ mothers.

The strength of the A antigen was determined by an immunohemolytic system which was previously deseribed in detail olsewhere [3]. Briefly, the technique consists of incubating a constant amount of a photometrically standardized cell suspension with a constant amount of hemolytic rabbit antihuman-A antibody and complement for 90 min at $37^{\circ} \mathrm{C}$. After centrifugation, the density of the hemoglobin in the surpenatant fluid is determined photometrically and the per cent hemolysis is taken as a measure of antigen strength. Fresh blood samples are essential for reproducible results. Although the subtype of A could generally be predicted from the hemolytic test, it was always verified with the lectins from Dolichos biflorus and Ulex europaeus, as well as human anti- $\mathrm{A}_{1}$. Two infants whose subtype was in doubt were retested at a later date.

The studies were carried out simultaneously with a population study being conducted on antigen strength among adults. This facilitated the experimental procedures because of the essential controls in the quantitative test.

The infants' sex, race, birth weight, and duration of gestation were ascertained from the records of the University Hospital only after all the laboratory tests wero completed.

\section{Results}

The distribution of the strength of 39 $A_{1}$ and $14 A_{2}$ cord blood samples are illustrated in Fig. 1, along with $289 A_{1}$ and $75 A_{2}$ "adult" individuals (6 years and older), tested in 1963 during a population study. The figure shows that antigen strength of cord cells is markedly weaker than that in adults. The mean antigen strength, expressed in percent hemolysis, of $39 \mathrm{~A}_{1}$ cord samples was $26.0 \pm 1.06$, while that of $289 \mathrm{~A}_{1}$ adults was $53.6 \pm 0.38$. The difference between the two is, of course, highly significant $(P<0.001)$. The mean for the $14 A_{2}$ cord cells was $4.2 \pm 0.72$ and that of 75 adult $A_{2}$ was $17.2 \pm 0.66$, a difference which is also highly significant $(P<0.001)$. Thus, the A antigen in both subtypes is weaker in cord cells than in corresponding adult subtypes. The strength of the $A_{1}$ antigen of cord cells is intermediate to the strength of the $A_{1}$ and $A_{2}$ of adults, but closer to $A_{2}$ with wide overlap in the values of cord $A_{1}$ and adult $A_{2}$. 


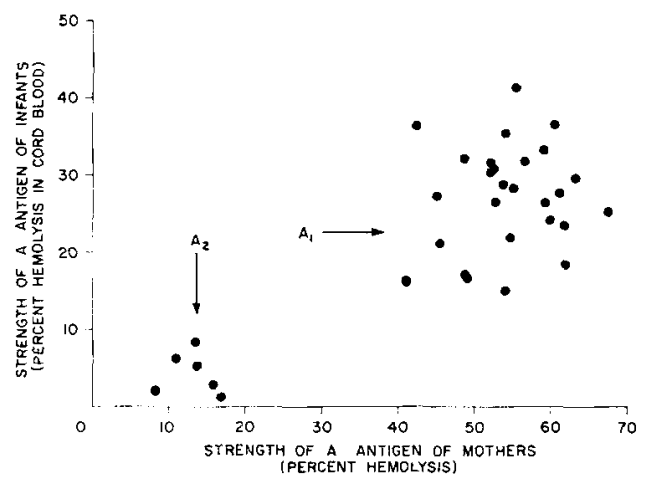

Fig. 2

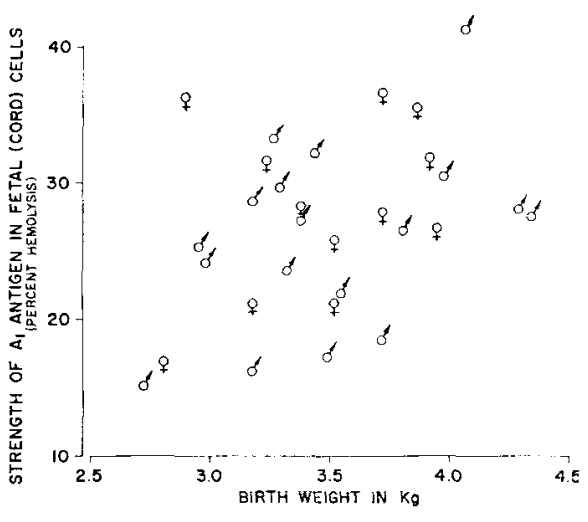

Fig. 3

Fig. 2. Relationship between antigen strength in the mother and that of her newborn infant.

Fig. 3. Relationship between antigen strength of newborn $A_{1}$ infants and birth weight.

Two $\mathrm{A}_{1} \mathrm{~B}$ cord blood samples gave hemolysis values of 8.9 and $11.0 \%$, respectively, but these are not included in the figure because the $\mathrm{A}$ antigen is known to be depressed in the presence of $B$.

Fig. 1 also reveals variation within the $A_{1}$ and $A_{2}$ subtypes both among adult and cord blood samples. The relative variation, expressed as the coefficient of variation, is $25 \%$ for the $A_{1}$ cord blood samples and $12 \%$ for the $A_{1}$ adults. Thus, variation relative to the mean is twice as large among $A_{1}$ cord blood samples than it is among $A_{1}$ adults.

The question arises as to the source(s) contributing to variation. The subtypes themselves are major sources of variation and have long been recognized as distinct genetic entities; therefore, the subtypes will be kept separate in the further analyses.

As stated previously, part of the variability in antigen strength within subtypes of type $A$ adults is inherited. This can be shown by parent-offspring relationships, as between the degree of dependency of the infants' antigen strength on that of its mother's. Thirty-three mother-infant pairs were available $\left(27 A_{1}\right.$ and $\left.6 A_{2}\right)$ in which both the mother and infant were of the same subtype. These comparisons are illustrated in Fig. 2. Numerically, the degree of dependence was calculated by regressing the infant's antigen strength on that of the mother. For $27 A_{1}$ motherinfant pairs, the regression coefficient was $b=0.13 \pm 0.21$ which, in light of the large standard error, is obviously not significant $(P>0.05)$. This coefficient means that for one unit increase in the mother's antigen strength, the infant's antigen strength increased 0.13 units. This low value indicates little association between the strength of the maternal $A_{1}$ and the fetal (cord) $A_{1}$. However, a portion of the infants are expected to have inherited the paternal $A_{1}$ and not that of the mother, which may partly be responsible for the low degree of mother-infant association. Nevertheless, it may be surmised that at the time of birth genetic factors contribute only a minor fraction to the total 
variation among $A_{1}$ infants. This finding prompted the search for other sources of variation, such as sex and birth weight.

Since the majority of infants were Caucasians and since racial differences contribute to differences in birth weight [7], only Caucasians were further analyzed. Occasionally, $\mathrm{A}_{2}$ mothers may form anti$A_{1}$ antibodies, therefore, no $A_{1}$ infants born to $A_{2}$ mothers were included. There were $30 A_{1}$ Caucasian infants born to $A_{1}$ mothers; 18 boys and 12 girls. They were all born at term except one girl who was, according to menstrual history, two weeks early.

The average antigen strength, expressed in percent hemolysis, was $25.9 \pm 1.57$ for boys and $28.4 \pm 1.85$ for girls. Although the average strength was slightly higher for girls than for boys, the difference is not significant $(P>0.05)$ due to the wide overlap in individual values and the relatively small numbers concerned.

The antigen strength according to birth weight, is shown in Fig. 3, where an association between birth weight and antigen strength is indicated. The magnitude of the association was determined by regression and correlation analysis. The computations were carried out separately for boys and girls because of the usual difference in birth weight. The regression equations were $Y=3.3 \mathrm{I}+6.45(X)$ for boys and $Y=9.31+5.49(\mathrm{X})$ for girls, where $X$ is the birth weight (in $\mathrm{kg}$ ) and $Y$ is the strength of the A antigen (expressed in per cent hemolysis). Each of the two regression coefficients was positive and approached significance at the $5 \%$ level. Since the slopes of the two regression lines were similar and not significantly different from each other $(P>0.30)$, the two sexes were combined by pooling the corrected sums of squares and the cross-products. The regression coefficient for the pooled values was $6.13 \pm 2.72$. This is significantly different from zero $(P<0.05)$, indicating that the association between birth weight and antigen strength is real and not due to chance. Hence, from the birth weights of infants born at term certain predictions can be made with respect to the strength of the $A_{1}$ antigen on fetal erythrocytes.

Similar results were obtained by correlating birth weight and antigen strength, the resulting coefficients being $r=0.43 \pm$ 0.24 for boys and $r=0.33$ for girls. The pooled correlation coefficient (pooled because they were not significantly different from each other) was $r=0.39 \pm 0.18$, which is significantly different from zero $(P<$ 0.05 ), again indicating an association between birth weight and strength of the fetal $A_{1}$ antigen.

The $A_{2}$ infants were not further analyzed because the number was relatively small and with the amounts of anti-A and complement used in the immunohemolytic system the percentage hemolysis of $\mathrm{A}_{2}$ infants was low, i.e., some values very close to the limit of detection.

\section{Discussion}

The results presented herein have disclosed that the A antigen in fetal (cord) erythrocytes is weaker than that in erythrocytes of adults. Newborn infants as well as adults manifest considerable variation in the strength of the $A$ antigen both between and within $A_{1}$ and $A_{2}$ subtypes. Although only type $A$ infants born to type A mothers were included in the present investigation, a similar degree of variation 
in antigen strength may also exist among infants born to type $O$ and $B$ mothers. This variation in antigen strength appears to be large enough to be of importance in the etiology of ABO hemolytic disease of the newborn because infants with relatively "strong" $A_{1}$ antigen appear more likely to have a portion of their red cells destroyed than infants with a "weaker" A antigen. Furthermore, the magnitude of difference in antigen strength between fetal and adult erythrocytes is seemingly large enough to constitute a major protective factor to the fetal red cells against maternal anti-A antibodies that have crossed the placental barrier.

The relatively "weak" A antigen and the variation in antigen strength among newborn infants may explain some peculiarities observed in ABO incompatibility, such as the observation of Gunson [6] that sensitization of infants' cells may occur without clinical manifestation of disease. In addition, the variation in the strength of the $A$ antigen at birth may explain in part the poor quantitative relationship between maternal antibody titer and of the ensuing hemolytic process. However, it is realized that other factors, such as the heterogeneity of antibodies, may also contribute to this poor quantitative relationship.

Variation in A antigen strength was previously reported by Fischer [2], but he apparently made no attempt to distinguish between $A_{1}$ and $A_{2}$ infants or to consider possible effects of antibodies in ABO incompatible pregnancies. With respect to the latter, type $A_{1}$ infants may display a relatively strong $A$ antigen because of partial sensitization of fetal erythrocytes by maternal anti-A antibodies.
On the other hand, in a marked hemolytic process the cells first destroyed are likely to have a relatively strong $A$ antigen; the remaining cells could display a relatively weak $A$ antigen. Thus, maternal antibodies could contribute to the observed variation in antigen strength among infants of $\mathrm{ABO}$ incompatible pregnancies.

Schellong [12] recently reported that premature infants rarely suffer from ABO hemolytic disease despite the presence of immune anti-A or anti-B antibodies. He interpreted this finding on the basis of "incomplete maturity" of the A and B antigens. Since the strength of the $A$ and $B$ antigens increases continuously during fetal life [8], the damage on fetal erythrocytes by maternal anti-A or anti-B is expected to increase with an increase in duration of pregnancy. Thus, in ABO incompatibility, destruction of fetal erythrocytes by maternal antibodies may be expected to occur predominantly in the latter stages of pregnancy and neonatally. This may explain the relatively high frequency of cases of ABO hemolytic disease characterized by hyperbilirubinemia and an absence of early anemia. On the other hand, severe early anemia is not an infrequent finding in $\mathrm{Rh}$ hemolytic disease. However, the $\mathrm{Rh}$ antigens are not known to undergo marked quantitative change during the individual's life and destruction of fetal erythrocytes by anti-Rh antibodies probably begins earlier than in $\mathrm{ABO}$ incompatible pregnancies.

Of the recognized sources of variation in antigen strength among $A_{1}$ infants born at term, birth weight showed the only significant effect. This effect was considerably larger than that of the maternal $A_{1}$ antigen. However, parental (or genetic) 
effects on variability are more pronounced among adult $A_{1}$ individuals than at birth as will be shown in a fortheoming publication. At birth, the genetic variation among $A_{1}$ individuals may become swamped by the more pronounced effect of birth weight.

The significant association between birth weight and strength of the $A_{1}$ antigen suggests that birth weight of infants born at term is more than an expression of size. To my knowledge this is the first instance where an association has been detected between a serological or biochemical characteristic and birth weight among infants born at term. The explanation of this association may be that strength of the $A_{1}$ antigen and birth weight both depend on the relative development of the infant born at term. Nevertheless, it is realized that birth weight is only a rough means of assessing relative development; birth weight is known to be influenced by various factors $[9,11]$. Relative strength of the $A_{1}$ antigen from embryo to approximately three years of age depends to a large part on the stage of development of the erythropoietic system and probably the relative development of the infant in general. Elsewhere [4] it was shown that the strength of the $A_{1}$ antigen increases very rapidly during the first few months after birth. Hence, it seems reasonable that well developed infants measured by birth weight at term are already exhibiting part of the rapid postnatal increase in antigen strength.

Infant girls displayed on the average a slightly stronger $A_{1}$ antigen than boys. This is in agreement with the notion that girls develop more rapidly (physiologic, skeletal) than boys of corresponding age, despite the fact that the boys exceed the girls in size and birth weight [14]. As suggested elsewhere [5], the difference in rate of development might explain the opposite effect of sex in physiologic jaundice and ABO hemolytic disease. An excess of boys was observed in physiologic jaundice $[1,13]$, while a highly significant excess of girls was found among the infants with ABO hemolytic disease [5]. However, a larger series of cord blood samples will have to be tested for establishing definitely a difference in antigen strength between the two sexes. Unfortunately, testing large numbers of cord blood samples by the immunohemolytic method presents some difficulties-fresh blood samples are required and the number of $A_{1}$ mothers who deliver $A_{1}$ infants is limited.

\section{Summary}

The quantitative aspects of the A antigen of fetal (cord) erythrocytes were investigated by an immunohemolytic system, utilizing 53 type $\mathrm{A}$ infants born to type A mothers. Cord cells were found to have a much weaker $A$ antigen than red cells of adults. Marked variation in the strength of the $A$ antigen was also found among and within the $A_{1}$ and $A_{2}$ subtypes. The variation among $A_{1}$ infants appeared to be large enough to be of importance in the etiology of ABO hemolytic disease of the newborn. The analysis of 30 cord blood samples of $A_{1}$ infants born at term to $A_{I}$ mothers revealed a significant association between the strength of the $A_{1}$ antigen and birth weight. The possible implications of these findings on ABO hemolytic disease and development were discussed. 


\section{Acknowledgements}

I wish to express my sincere appreciation to Dr. T. N. Evans and the staff of the Maternity Clinic for collecting the cord blood samples. I am also indebted to Dr. David E. Anderson for his valuable assistance with the manuscript and to Dr. Henry Ger- showitz and Dr. Donald C. Shreffler for making available their blood typing records. A portion of the samples used in the control population was made available by the Cardiovascular Research Center, The University of Michigan, supported by program project grant H-6378 from the National Heart Institute.

\section{References}

1. Crosse, V. M., Mwyer, T. C. and GerRard, J. W.: Kernicterus and prematurity. Arch Dis Child, 30: 501, 1955.

2. Fischer, K.: Morbus haemolyticus neonatorum im A BO-System, p. 55. Thieme, Stuttgart, 1961 .

3. Grundbacher, F. J.: Immunohemolysis as a method for quantitation of the $A$ antigen of human erythrocytes. $J$ Immunol, 93: 205, 1964.

4. - Changes in the human $A$ antigen of erythrocytes with the individual's age. Nature (Lond), 204: 192, 1964.

5. - ABO hemolytic disease of the newborn: a family study with emphasis on the strength of the A antigen. Pediatrics, 35: 916, 1965.

6. Gunsox, H. H.: Symposium on haemolytic disease of the newborn with special reference to ABO incompatibility. Some serological aspects. Proc Royal Soc Med, 56: $154,1963$.

7. Holman, G. H.: Studies on physiologic hyperbilirubinemia of Negro and White premature infants. Pedialrics, 22:1115, 1958.

8. Lodenkämper, H. and StTEnen, G.: Über die Bindungsfähigkeit der roten Blutkörperchen für die Isoagglutinine in den einzelnen Lebensabschnitten. Aerztl Wschr, 8:110, 1953.

Submitted Feb. 8, 1965

Accepted Feb. 17, 1965
9. Prenose, L. S.: Some recent trends in human genetics, in Proceedings 9th International Congress of Genetics (Bellagio), Pt. 1 (Suppl to Caryologia), Volume 6, p. 521, 1954.

10. Polley, M. J., Adinolfi, M. and Mollison, P. I.: Serological characteristics of anti-A related to type of antibody protein $(7 \mathrm{~S} \gamma$ or 19Sy). Vox Sang, 8: 385, 1963.

11 Robson, E. B.: Birth weight in cousins. Ann Hum Genet, 19: 262, 1955.

12. Schellong, G.: Ủber den Einfluss mütterlicher Antikörper des ABO-Systems auf die Reticulocytenzahl und Serumbilirubin bei Frühgeborenen. $Z$ Kinderheilk, 90:134, 1964.

13. Schneewriss, B. and Wiegland, U.: Klinische Untersuchungen bei Hyperbilirubinämien im Neugeborenalter. I. Mitteilung. Z Kinderheilk, 89:277, 1964.

14. Tanner, J. M.: Growth at Adolescence, 2nd ed., p. 325, Blackwell Scientific Publications, Oxford, 1961.

15. Zuelzer, W. W and Kaplan, E.: ABO heterospecific pregnancy and hemolytic disease: a study of normal and pathologic variants. IV. Pathologic variants. Amer $J$ Dis Child, 88: $319,1954$.

Department of Human Genetics The University of Michigan Medical School Ann Arbor, Michigan 48104 U.S.A. 